\title{
Non-enzymatic Colorimetric Glucose Detection Based on Au/Ag Nanoparticles Using Smartphone and Machine Learning
}

Volkan KILIÇ, ${ }^{* \dagger}$ Öykü B. MERCAN, ${ }^{*}$ Mehmet TETİK, ** Özlem KAP, *** and Nesrin HORZUM***

* Department of Electrical and Electronics Engineering, Izmir Katip Celebi University, Izmir, 35620, Turkey

** Department of Biocomposite Engineering, Graduate School of Natural and Applied Sciences, Izmir Katip Celebi University, Izmir, 35620, Turkey

*** Department of Engineering Sciences, İzmir Katip Çelebi University, 35620 Turkey

$\dagger$ To whom correspondence should be addressed.

E-mail: volkan.kilic@ikcu.edu.tr 


\begin{abstract}
Conventional enzyme-based glucose quantification approaches are not feasible due to their high cost, specific working temperatures, short shelf life, and poor stability. Therefore, a portable platform, which offers rapid response, cost-efficiency, and high sensitivity, is indispensable for the healthcare of diabetes. In this study, we proposed a portable platform incorporating gold $(\mathrm{Au})$ and silver $(\mathrm{Ag})$ nanoparticles $(\mathrm{NPs})$ with a smartphone application based on machine learning for non-enzymatic glucose quantification. The color change obtained from the reaction of small and large $\mathrm{Au} / \mathrm{Ag}$ NPs with glucose was captured using a smartphone camera to create a dataset for the training of machine learning classifiers. Our custom-designed user-friendly smartphone application called "GlucoQuantifier" uses a cloud system to communicate with a remote server running a machine learning classifier. Among the tested classifiers, linear discriminant analysis exhibits the best classification performance (93.63\%) with small $\mathrm{Au} / \mathrm{Ag}$ NPs which demonstrates that incorporating $\mathrm{Au} / \mathrm{Ag}$ NPs with machine learning under a smartphone application can be used for the non-enzymatic glucose quantification.
\end{abstract}




\section{Introduction}

The diabetes epidemic will affect hundreds of millions of people in the coming years, ${ }^{1,2}$ and the diagnostic remains as vital as it was in the past. Diabetes-related complications include both microvascular and macrovascular diseases that can damage the retina, skin, kidney, small and large vessels, central and peripheral nerves. Blood glucose is crucial in the monitoring of diabetes due to its potential as a biomarker. When the glucose level is routinely monitored and regulated in diabetics, complications can be avoided or at least delayed. Several systems, therefore, have been proposed to measure glucose which can be categorized into two classes: namely, optical and electrochemical biosensors. ${ }^{3}$

Optical systems calculate glucose levels based on the measurement of photons. ${ }^{4}$ Representative methods in this category are absorptiometry, ${ }^{5}$ reflectometry, ${ }^{6}$ fluorescence $^{7}$ and surface plasmon resonance. ${ }^{8}$ Electrochemical biosensors perform glucose measurement based on voltammetric, amperometric, or potentiometric methods which can be classified into enzymatic or non-enzymatic approaches regarding the presence of an enzyme in the measurement process. Glucose oxidase (GOx) is the most commonly used enzyme that catalyzes the oxidation of glucose into gluconic acid with high specificity producing hydrogen peroxide $\left(\mathrm{H}_{2} \mathrm{O}_{2}\right)$ as a by-product. Hereby, the glucose concentration is measured by the amount of $\mathrm{H}_{2} \mathrm{O}_{2}$ production or $\mathrm{O}_{2}$ consumption during the oxidation reactions. However, enzyme-based glucose detection methods have drawbacks such as high cost, specific working temperatures, short shelf life, and poor stability. Therefore, as an alternative to enzyme-based methods, non-enzymatic methods are popular for glucose detection because of their low-cost, rapid response, and high sensitivity. For non-enzymatic detection, rapid advances in nanoscience and nanotechnology have led to the emergence of various nanostructures including non- 
biological electrode materials which have been gaining relevance due to their cost efficiency, stability, robustness, and having a tunable catalytic activity over the enzymes. Non-biological electrodes like metal oxide nanostructures $\left(\mathrm{NiO},{ }^{9} \mathrm{CuO},{ }^{10} \mathrm{ZnO},{ }^{11} \mathrm{MnO}_{2},{ }^{12}\right.$ $\left.\mathrm{CeO}_{2},{ }^{13} \mathrm{Co}_{3} \mathrm{O}_{4},{ }^{14} \mathrm{CoMoO}_{4},{ }^{15} \mathrm{MnCaO}_{2},{ }^{16}\right)$ noble metal nanostructures $\left(\mathrm{Au},{ }^{17} \mathrm{Ag}^{18}\right)$, transition metal dichalcogenides $\left(\mathrm{MoS}_{2},{ }^{19} \mathrm{WSe}_{2},{ }^{20} \mathrm{WS}_{2}{ }^{21}\right)$, carbon-based nanomaterials (graphene, ${ }^{22}$ graphene oxide, ${ }^{23}$ carbon dots, ${ }^{24}$ carbon nanotubes ${ }^{25}$ ), layered double hydroxides, ${ }^{26,27}$ and magnetic nanoparticles $\left(\mathrm{MFe}_{2} \mathrm{O}_{4}(\mathrm{M}=\mathrm{Mg}, \mathrm{Ni}, \mathrm{Cu}),{ }^{28} \mathrm{Fe}_{3} \mathrm{~S}_{4},{ }^{29}\right.$ $\mathrm{Fe}_{3} \mathrm{O}_{4}{ }^{30}$ ) have been used as nanozymes mimicking the enzyme activity. Among the nanostructures, silver nanoparticles (Ag NPs) and gold nanoparticles (Au NPs) offer advantages in biosensor applications due to their easy preparation, unique electrical and optical properties, and controllable size and stability which affect the sensitivity and selectivity of detection. ${ }^{31,32} \mathrm{Au}$ NPs and Ag NPs have characteristic optical properties in the visible region depending on their dispersion and aggregation in a liquid. ${ }^{33,34}$ Therefore, the interaction between the NPs and glucose molecules leads to a color change, resulting in glucose level measurements with colorimetric approaches. Li et al. reported a nonenzymatic method using gold nanostructures for glucose level detection forming a silver film via the silver mirror reaction (SMR). ${ }^{17}$ The Au NPs assisted SMR results in a coreshell (Au-Ag) structure which can be verified with surface plasmon resonance by checking disappearance and appearance peaks at $520 \mathrm{~nm}$ and $410 \mathrm{~nm}$ for $\mathrm{Au}$ and $\mathrm{Ag}$, respectively. Depending on the size and surface charge of the NPs, the color of the solution turns from red to yellow which allows the colorimetric analysis. The effect of the surface charge has been studied for $\mathrm{Au}$ and $\mathrm{Ag}$ nanostructures on colorimetric detection by synthesizing negatively charged polystyrene sulfonate-coated Au nanorods (PSS-Au NRs), citrate capped gold nanospheres (C-Au NSs), and positively charged cetyltrimethyl ammonium bromide capped Au NRs, quaternary ammonium capped gold nanospheres. ${ }^{18}$ 
The surface charge-dependent color change was attributed to the generation of Ag NPs which allows the redox reaction between $\mathrm{Ag}^{+}$and glucose around the negatively charged PSS-Au NRs and C-Au NSs. Recently, Gao et al. have conducted colorimetric glucose detection by producing gluconic acid and $\mathrm{H}_{2} \mathrm{O}_{2}$ in the presence of oxygen using $\mathrm{Au}$ NPs as a catalyst. ${ }^{35}$ Subsequently, Ag NPs were added to the solution in which Ag NPs is etched with $\mathrm{H}_{2} \mathrm{O}_{2}$ produced in different concentrations. The yellow color of the Ag NPs either gradually faded, or the Ag NPs were entirely dissolved in $\mathrm{H}_{2} \mathrm{O}_{2}$, and the solution has turned completely red, due to the presence of $\mathrm{Au}$ NPs. This method enabled determining the glucose concentration based on a color scale. The selectivity was enhanced by using L-cysteine. ${ }^{36}$

Furthermore, boronic acid derivatives are being used in glucose detection, due to their reversible binding ability with the glucose to form cyclic boronate esters. ${ }^{37}$ A nonenzymatic method for colorimetric glucose determination was performed using Au NPs capped with 4-cyanophenyl boronic acid incorporated with $\beta$-cyclodextrin. An observable color change from red to blue with increasing glucose concentration was explained by the aggregation of the functionalized Au NPs due to the analyte induced inter plasmon coupling. Another example of the use of boronic acid derivative in glucose detection was reported by Li et al. who prepared 3-aminophenyl boronic acid-modified Au NPs by the citrate reduction. ${ }^{38}$ UV-Vis spectra results showed that with increasing the glucose concentration, the peak at $520 \mathrm{~nm}$ was disappeared, and the peak at $650 \mathrm{~nm}$ was increased. This was explained by the fact that a higher glucose concentration prevents aggregation of $\mathrm{Au}$ NPs.

In the colorimetric analysis, various color spaces such as RGB (Red-GreenBlue), HSV (Hue-Saturation-Value) and L*a*b* (Lightness, Green-Red, Blue-Yellow) can be used to extract the color information for glucose measurement. ${ }^{39-42}$ Color spaces 
were employed depends on the applications. The HSV color space was used in alcohol detection ${ }^{43}$ while the $\mathrm{L}^{*} \mathrm{a}^{*} \mathrm{~b}^{*}$ was performed in $\mathrm{pH}$ measurement. ${ }^{44}$ On the other hand, the RGB was employed for the detection of chlorine in water ${ }^{45}$ and ripeness estimation of fruits. ${ }^{46}$ In the aforementioned studies, color space parameters were used to derive an analytical expression for qualitative and quantitative evaluations. However, camera optics and ambient light conditions have adverse effects on colorimetric analysis. Advanced algorithms like machine learning have been employed in the colorimetric evaluation process to overcome these problems. ${ }^{47,48}$ The machine learning algorithms have powerful utilities like automated decision-making and self-learning from the data. The advantage of machine learning algorithms lies in their flexibility and adaptability to new platforms such as smartphone-based systems. Recent advances in smartphone technology lead to emerge of many platforms capable of running sophisticated algorithms for sensitive and reliable colorimetric analysis. SPAQ application was developed to use histogram distribution for the detection of alcohol levels in saliva. ${ }^{43,49}$ Colorimetric Test Reader ${ }^{50}$ application was used to detect $\mathrm{pH}$, protein, and glucose values in an assay while the Colorimetric Plate Reader application was developed for ELISA tests. ${ }^{51}$ The univariate and multivariate analyses were run in PhotoMetrix ${ }^{52}$ to quantify the analytes while fuzzy classifier was used in FuzzyChem application for peroxide quantification. ${ }^{53}$ In GlucoSensing application, images, from the gallery or captured by the smartphone camera, were sent to the remote server which runs machine learning classifiers for colorimetric quantification of glucose concentrations. ${ }^{48}$

Here, we develop an application-based solution to colorimetric testing of glucose using machine learning classifiers. This work demonstrates the advantageous combination of a smartphone-based non-enzymatic glucose sensing system and machinelearning algorithms for the first time. The effect of different-sized Au NPs and Ag NPs as 
the GOx mimic on glucose detection was investigated. After the classifiers were trained with the dataset created with the images captured under white LEDs illumination condition, they were run in the remoter server that can be accessed via the cloud service by our custom-designed Android application with a user-friendly interface that even nonexpert users may use without extensive training. The proposed methodology incorporating machine learning classifiers to quantify glucose concentration shows great promise in supplying the ultimate solution to smartphone-based colorimetric sensing.

\section{Materials and Methods}

\section{Materials}

Hydrogen tetrachloroaurate (III) trihydrate $\left(\mathrm{HAuCl}_{4} \cdot 3 \mathrm{H}_{2} \mathrm{O}, 99 \%\right)$ was purchased from Alfa Aesar; trisodium citrate dihydrate $\left(\mathrm{Na}_{3} \mathrm{C}_{6} \mathrm{H}_{5} \mathrm{O}_{7} \cdot 2 \mathrm{H}_{2} \mathrm{O}, \geq 99 \%\right)$, poly(vinyl pyrrolidone) (PVP; Mw: $10 \mathrm{~kg} / \mathrm{mol})$, silver nitrate $\left(\mathrm{AgNO}_{3}, 99 \%\right)$, sodium borohydride $\left(\mathrm{NaBH}_{4}, \geq 98 \%\right)$, L-cysteine $\left(\mathrm{C}_{3} \mathrm{H}_{7} \mathrm{NO}_{2} \mathrm{~S}, 97 \%\right)$, starch $\left(\left(\mathrm{C}_{6} \mathrm{H}_{10} \mathrm{O}_{5}\right)\right.$ n), D-galactose, $(\geq 98 \%)$, D-glucose, $(\geq 99.5 \%)$, D-maltose monohydrate, $(\geq 99 \%)$, and D-xylose, $(\geq 99 \%)$ were purchased from Sigma-Aldrich. All chemicals were analytical reagent grade and used as received. Ultrapure water $\left(18.2 \mathrm{M} \Omega \cdot \mathrm{cm}^{-1}\right.$ at $\left.25^{\circ} \mathrm{C}\right)$ used in all experiments was produced by a Human Corporation water treatment system. All glassware was initially cleaned with detergent and thoroughly rinsed with the water prior to use.

\section{Synthesis of NPS}

Synthesis of Au NPs: The colloidal dispersions of different-sized Au NPs were prepared by the reduction of $\mathrm{HAuCl}_{4}$ with $\mathrm{NaBH}_{4}$ using $\mathrm{Na}_{3} \mathrm{C}_{6} \mathrm{H}_{5} \mathrm{O}_{7}$ as a stabilizer at ambient temperature ${ }^{35}$ and the traditional Turkevich method ${ }^{54}$ using PVP as a stabilizer. ${ }^{55}$ In the 
former method, aqueous solutions of $\mathrm{HAuCl}_{4}(10 \mathrm{mM}, 0.5 \mathrm{~mL})$ and $\mathrm{Na}_{3} \mathrm{C}_{6} \mathrm{H}_{5} \mathrm{O}_{7}(10 \mathrm{mM}$, $0.5 \mathrm{~mL}$ ) were mixed and completed to a total volume of $20 \mathrm{~mL}$. Then freshly prepared $\mathrm{NaBH}_{4}(1.0 \mathrm{mM}, 0.5 \mathrm{~mL})$ was added into the solution under magnetic stirring at ambient temperature. The solution color turned immediately from pale yellow to cherry-red. Lcysteine solution $(0.1 \mathrm{mM}, 0.4 \mathrm{~mL})$ was added into the Au NPs dispersion to stabilize the colloids and the final solution was stored at $4{ }^{\circ} \mathrm{C}$. In the latter one, after loading an aqueous $\mathrm{HAuCl}_{4}$ solution $(0.24 \mathrm{mM}, 200 \mathrm{~mL})$ into a single-neck flask and heating to reflux by magnetic stirring, a solution of $\mathrm{Na}_{3} \mathrm{C}_{6} \mathrm{H}_{5} \mathrm{O}_{7}(0.34 \mathrm{M}, 1.0 \mathrm{~mL})$ was added to the flask. The solution was refluxed for 30 minutes and its color changed from pale yellow to light cherry-red. The resulting mixture was centrifuged at $6000 \mathrm{rpm}$ for $1 \mathrm{~h}$ and redispersed in $2.0 \mathrm{~mL}$ of water, then PVP solution $(10 \mu \mathrm{L}, 0.166 \mathrm{mM})$ was added into the dispersion to stabilize the colloids. The final solution was also stored at $4{ }^{\circ} \mathrm{C}$.

Synthesis of Ag NPs: Ag NPs were synthesized using $\mathrm{NaBH}_{4}$ as a reducing agent, $\mathrm{Na}_{3} \mathrm{C}_{6} \mathrm{H}_{5} \mathrm{O}_{7}{ }^{35}$ and $\operatorname{starch}^{56}$ as stabilizers. In the first method, aqueous solutions of $\mathrm{AgNO}_{3}$ (10 mM, $0.5 \mathrm{~mL})$ and $\mathrm{Na}_{3} \mathrm{C}_{6} \mathrm{H}_{5} \mathrm{O}_{7}(40 \mathrm{mM}, 0.5 \mathrm{~mL})$ were mixed and filled with water to a total volume of $20 \mathrm{~mL}$. A freshly prepared $\mathrm{NaBH}_{4}(1.0 \mathrm{mM}, 0.5 \mathrm{~mL})$ was added to the solution under magnetic stirring in an ice-water bath. The colorless solution turned yellow. Ag NPs in colloidal dispersion were stabilized by L-cysteine solution $(0.1 \mathrm{mM}, 0.4 \mathrm{~mL})$ and the final solution was stored at $4{ }^{\circ} \mathrm{C}$. In the second method, the starch solution was prepared as $2 \%(\mathrm{w} / \mathrm{v})$ in boiling water and was used after cooling down to room temperature. $0.063 \mathrm{~g} \mathrm{AgNO}_{3}$ and $0.021 \mathrm{~g} \mathrm{NaBH}_{4}$ were separately dissolved in $50 \mathrm{~mL}$ of the starch solution. Then, the $\mathrm{AgNO}_{3}$ solution was added drop-by-drop into the $\mathrm{NaBH}_{4}$ solution at an estimated rate of $0.2 \mathrm{~mL} / \mathrm{s}$ under magnetic stirring. The solution color turned immediately from colorless to dark brown. The total volume of the colloid was 
diluted with the water to be $130 \mathrm{~mL}$ and allowed to boil for an additional 30 minutes. The colloid was then cooled to room temperature and aged for $12 \mathrm{~h}$ to eliminate the residual $\mathrm{NaBH}_{4}$. The final volume of Ag NPs colloid was diluted 1:10 and used within $24 \mathrm{~h}$.

Characterization of the NPs: UV-Vis spectroscopy measurements were carried out using an Ocean Optics HR2000 fiber optic spectrometer (FL, USA). The morphological characterization of the NPs was carried out by scanning electron microscopy (SEM) at accelerating voltages of $3.00 \mathrm{kV}$ and working distances of 1.7-3.9 $\mathrm{mm}$ in a Carl Zeiss 300 VP microscope equipped with an energy dispersive detector. Colloids for SEM observation were prepared by dropping the diluted (1:10 in water) dispersions on small silicon wafers that were subsequently dried. The diameter of the NPs was measured over the SEM micrographs using Fiji-Image $J$ software. The NPs obtained using $\mathrm{Na}_{3} \mathrm{C}_{6} \mathrm{H}_{5} \mathrm{O}_{7}$ as stabilizers are named as large NPs ( $\ell$-Au NPs) and ( $\ell$-Ag NPs) while the others are named as small NPs ( $s$-Au NPs) and ( $s$-Ag NPs). Table 1 reports the synthesized NPs employed in this work with the corresponding particle sizes.

\section{Experimental design and image capturing}

The different volume ratios of $\mathrm{Au}$ and $\mathrm{Ag}$ nanoparticle dispersions were investigated through the absorbance mFeasurements to determine the appropriate amount for the sensing experiments. A series of $200 \mu \mathrm{L}$ of Au NPs solution was added into the $700 \mu \mathrm{L}$ of saturated water with oxygen in glass vials. Then, $300 \mu \mathrm{L}$ of glucose solution with different concentrations $(0.5,1.0,2.0,3.0,4.0,5.0,6.0,7.0,8.0$, and $9.0 \mathrm{mM})$ was spiked and kept for 5 minutes. Subsequently, $800 \mu \mathrm{L}$ of Ag NPs solution was added into the mixture and incubated for $1 \mathrm{~h}$. Each solution was taken into the disposable cuvette and placed in a bench-type cardboard photo studio $(40 \times 40 \times 25 \mathrm{~cm})$ as illustrated in Fig. 1(a). 
Images were captured in the cardboard photo studio that was painted white inside and black outside to minimize ambient light conditions. White LEDs were placed inside on the cardboard ceiling $22.5 \mathrm{~cm}$ over the samples for illumination. The images were captured using a smartphone camera (LG G6, 1/3.06 inc. sensor size with $1440 \times 2880$ resolution, $1.12 \mu \mathrm{m}$ pixel size) with a tripod located $11.5 \mathrm{~cm}$ away from the samples. Fig. 1(b) shows the solutions with different glucose concentration levels ranging from 0 to 9.0 $\mathrm{mM}$. Ten consecutive images were captured for each concentration which leads to 110 images for the dataset. This dataset was transferred to a computer for image processing and feature extraction in MATLAB (MathWorks, MA, USA) environment. The extracted features were then used in the training of machine learning classifiers in Python software.

\section{Machine learning classifiers}

Machine learning classifiers were trained to detect concentration levels of glucose in solutions based on color change and their performances were evaluated with classification accuracy scores. Linear discriminant analysis (LDA), ${ }^{57}$ bagging classifier (BC), ${ }^{58}$ and random forest $(\mathrm{RF})^{59}$ classifiers showed the best performances in terms of the ability to detect glucose concentration.

The LDA classifier uses the Bayesian rule to estimates the mean and variance from the data for each class. Ensemble learning methods that use several classifiers and aggregate their results are frequently preferred in machine learning. ${ }^{60}$ Ensemble learning methods are generally distinguished as bagging and boosting. $\mathrm{BC}$ is one of the ensemble learning methods which aggregates individual predictions by voting or averaging to obtain the final prediction. RF is also a kind of ensemble method. In standard trees, each node is divided using the best split among all variables, while in the random forest approach, each node is split using the best of a subset of randomly selected predictors at 
that node. Therefore, the random forest classifier is more resistant to overfitting and uses only two variables: the number of variables in the random subset and the number of trees in the forest.

To train the machine learning classifiers, the images in the dataset were captured using a smartphone camera in JPEG format with a size of $4160 \times 3120$. Although the size of the images is large, solutions occupy a small region in the image. The region of interest (ROI), which is the area of the color change, is cropped for feature extraction. In this study, color and texture features were extracted from the ROI to classify the concentration level of solutions with machine learning classifiers. Firstly, RGB values of the image were converted to HSV and $\mathrm{L}^{*} \mathrm{a}^{*} \mathrm{~b}^{*}$ to analyze the effect of color spaces on the concentration level. Then, mean, skewness and kurtosis values were calculated for R, G, B, H, S, V, L*, a*, b* color channels. Thus, 27 color features were obtained.

After color features were extracted from the ROI of images, texture features were also extracted to increase the accuracy of machine learning classifiers. Texture features are statistical features obtained based on the intensity and color transition of images. Gray Level Co-Occurrence Matrix is one of the methods that is commonly used to extract features from texture analysis. Contrast, correlation, homogeneity and energy values of images were extracted as texture features. In addition to color and texture features, entropy and intensity values of the ROI were calculated. Totally, 33 extracted features for each of the 110 images in the dataset will be used in the training of machine learning classifiers. Representations of features with heatmap styles are given in supporting information (Figs. S1, S2 and S3).

$\mathrm{K}$-fold cross-validation is an important technique that is used to evaluate the performance of classifiers. ${ }^{61}$ In the K-fold technique, a dataset is split into K equal folds. K-1 folds are used for training while the remaining fold is used for testing. This process 
is repeated $\mathrm{K}$ times until each fold is used as test data. Thus, $\mathrm{K}$ different accuracy values are obtained. The average of these values is calculated to evaluate the performance of classifiers. In this study, $\mathrm{K}$ is set to 10 as it was found to be adequate to avoid high bias and variance problems. ${ }^{48,62}$

\section{Smartphone application: GlucoQuantifier}

In the previous subsection, machine learning classifiers were trained and compared based on their performance. Here, we developed a custom-designed smartphone application, named GlucoQuantifier, that allows the users to quantify the glucose concentration using a smartphone camera. With a simple and user-friendly interface, GlucoQuantifier is capable of transferring images from a smartphone to a remote server that runs a machine learning classifier. To set communication between the smartphone and remote server, the Firebase cloud system is used as it supports both Android (smartphone) and Python (remote server).

Among the tested classifiers, the LDA classifier was integrated with the GlucoQuantifier to quantify the glucose concentration of a new image as it outperformed the other classifiers in the training stage. When the user runs GlucoQuantifier to quantify the glucose concentration of the solution, the first page lets the user capture a new image using the smartphone camera or select an image from the gallery as shown in Fig. 2(a). After the image is selected, the colored area is cropped using an adjustable cropbox (Fig. 2(b) and (c)). Then the user needs to tap the upload button to send the cropped image to the server (Fig. 2(d)). The LDA classifier in the server runs to decide the class of glucose concentration based on extracted features (Fig. 2(e)). The result comes back to the GlucoQuantifier via the Firebase and it is displayed on the screen as shown in Fig. 2(f). 


\section{Experimental Evaluation}

The objective of this work is to investigate the effect of Au and Ag NPs diameters on nonenzymatic glucose detection using machine-learning algorithms. This section presents experimental evaluations of the proposed system for colorimetric quantification of glucose. We start with a discussion of different-sized descriptions of the Au and Ag NPs, before giving the analysis and comparison of the results.

\section{Characterization of different-sized Au and Ag NPs}

In general, Au NPs can catalyze the oxidation of glucose to gluconic acid in the presence of oxygen, producing $\mathrm{H}_{2} \mathrm{O}_{2}$ as a by-product. When $\mathrm{Ag}$ NPs are added to the solution, the generated $\mathrm{H}_{2} \mathrm{O}_{2}$ etches the yellow-colored $\mathrm{Ag}$ NPs to colorless silver ions and the red color of Au NPs appears. Therefore, Au/Ag NPs system enables a colorimetric glucose determination without requiring any enzymes or chromogenic reagents. ${ }^{35} \mathrm{Au}$ and $\mathrm{Ag}$ NPs were synthesized by the $\mathrm{NaBH}_{4}$ reduction of gold and silver salts, respectively. Sodium citrate was used as a stabilizer at room temperature. To obtain smaller and more stable NPs, the syntheses were performed under heat-up conditions and using PVP and starch as the polymeric stabilizers. Increasing the reaction temperature not only provides the rapid reduction of $\mathrm{Au}$ or $\mathrm{Ag}$ ions but also their homogeneous nucleation afterward, allowing the formation of small-sized NPs. ${ }^{63,64}$ PVP and starch act as surfactants and the size controllers for Au and Ag NPs, respectively. Stabilization of the smaller NPs occurs through the interactions between the NPs and the hydrophilic groups of PVP (carbonyl oxygen and nitrogen of the repeating unit) and starch (ether oxygen and free hydroxyl), thus preventing aggregation. ${ }^{56,65}$

The UV-Vis absorption spectra of the Au NPs, Ag NPs, and Au/Ag NPs are 
given in Fig. 3(a) and (b). While the spectrum of the Au NPs has an absorption maximum nearly at $530 \mathrm{~nm}$; the spectrum of the Ag NPs has an absorption maximum nearly at 405 nm. Au/Ag NPs mixtures were prepared using different volume ratios of Au to Ag NPs. An appropriate volume ratio of Au to Ag NPs was selected as 1:4 through the absorbance measurements. The SEM micrographs presented in Fig. 3 (c) and (d) demonstrate that both Au and Ag NPs are spherical-shaped but agglomerated. The histograms in Fig. 3 (e) and (f) show the particle size distributions of the Au and Ag NPs, respectively. The average particle sizes calculated based on the SEM micrographs were $30 \pm 12 \mathrm{~nm}(\ell-\mathrm{Au}$ NPs) and $25 \pm 5 \mathrm{~nm}(\ell-\mathrm{Ag}$ NPs), respectively (see also in Table 1).

Fig. 4(a) shows the absorption spectrum for the smaller Au and Ag NPs. The sharper absorption bands occurring at wavelengths of $520 \mathrm{~nm}$ and $400 \mathrm{~nm}$ arise from $\mathrm{Au}$ and Ag NPs, respectively. The absorption maxima are expected to shift towards the blue wavelength, higher frequency, and energies as a result of the increment in electron density of the particles when the particles decrease in size. ${ }^{66,67}$ SEM micrographs of the smaller $\mathrm{Au}$ and Ag NPs are demonstrated in Fig. 4 (b) and (c), respectively. Monodispersed spherical NPs were obtained with the narrower size distribution, as shown in Fig. 4 (d) and (e). The average particle size of $s$-Au NPs was $16 \pm 4 \mathrm{~nm}$, while $s$-Ag NPs was $12 \pm 2$ $\mathrm{nm}$ (see also in Table 1).

$\ell$ - and $s$-Au/Ag NPs as colorimetric probes for enzyme-free detection of glucose were used without $\mathrm{pH}$ control. The glucose concentrations were in the range of 0.5-9.0 mM. The images of color changes with the increasing glucose concentrations were captured using a smartphone camera. Au NPs acted as a catalyst in the Au/Ag NPs system, and color change was obtained by the etching of the $\mathrm{Ag}$ NPs by $\mathrm{H}_{2} \mathrm{O}_{2}$ produced as a byproduct. It should be noted that surface charges play an essential role in the aggregation of the NPs ${ }^{68}$ NPs with negative surface charges are critical in colorimetric determination 
for electrostatic stabilization. ${ }^{69}$ Especially in this study, the etching of the aggregated Ag NPs by $\mathrm{H}_{2} \mathrm{O}_{2}$ becomes difficult, and the color change would be subtle for sensitive colorimetric detection. As seen on the SEM micrographs, $\ell$-Ag NPs are aggregated more than s-Ag NPs in Fig. 3(d) and Fig. 4(c), respectively. Hence, the color change sensitivity was lower to determine the glucose concentration accurately for $\ell-\mathrm{Au} / \mathrm{Ag}$ NPs. Moreover, $s$-Au NPs contributed to the accuracy of the colorimetric detection due to their smaller size and less aggregation compared to the $\ell$-Au NPs. ${ }^{70}$

\section{Machine learning-based colorimetric glucose detection}

Color and texture features were extracted as described in Machine learning classifiers. First, images were cropped to obtain ROI. Then 33 features were extracted from ROI to train machine learning classifiers.

The performance of LDA, BC, and RF classifiers on $\ell-\mathrm{Au} / \mathrm{Ag}$ NPs and $s-\mathrm{Au} / \mathrm{Ag}$ NPs was given in Table 2. They show that each classifier showed higher performance for $s$-Au/Ag NPs, also classifier performance improved for solutions on the $2^{\text {nd }}$ day. The LDA has shown the highest accuracy in $s$-Au/Ag NPs with $93.63 \%$ and $\ell-\mathrm{Au} / \mathrm{Ag}$ NPs with $90.00 \%$ on the second day.

Accuracy (Eq. (1)), precision (Eq. (2)), recall (Eq. (3)) and f1-score (Eq. (4)) metrics were used to evaluate the performance of the machine learning classifiers.

$$
\begin{aligned}
& \text { Accuracy }=\frac{T P+T N}{T P+T N+F P+F N} \\
& \text { Precision }=\frac{T P}{T P+F P} \\
& \text { Recall }=\frac{T P}{T P+F N}
\end{aligned}
$$




$$
f 1 \text { score }=2 \times \frac{\text { Precision } \times \text { Recall }}{\text { Precision }+ \text { Recall }}
$$

True positive and true negative means that a model correctly predicts the positive and negative class, while false positive and false negative means that a model incorrectly predicts the positive and negative class. Accuracy gives a ratio of the number of correct predictions to the total number of predictions. Precision is defined ratio of true positive predictions to all positive predictions while recall is a ratio of true positive predictions to total true positives and false negatives. $\mathrm{fl}$-score is calculated by the harmonic average of precision and recall and it has values in the range of 0 and 1 . The average score of precision, recall, and $\mathrm{fl}$ metrics for the LDA classifier of the $2^{\text {nd }}$ day on $\ell-\mathrm{Au} / \mathrm{Ag}$ NPs and $s-\mathrm{Au} / \mathrm{Ag}$ NPs are shown in Table 3 . Tables for the $1^{\text {st }}$ day are given in the Supplementary data (Tables S1 and S2). The performance of the LDA classifier on the $2^{\text {nd }}$ day was also evaluated with confusion matrices which were given in Fig. 5 for both datasets where "True Label" defines the actual class while "Predicted Label" indicates estimated class by the classifier. Each row of the confusion matrix corresponds to an estimated class for the respective actual class. Confusion matrices of the LDA classifier on the $1^{\text {st }}$ day are given in the Supplementary data (Figs. S4 and S5). The confusion matrix is used to evaluate the performance of classifiers. It compares the true label to the predicted label then visualizes the accuracy of the classifier. In Fig. 5(a) and (b), diagonal elements represent the number of predicted correctly samples. In Fig. 5(a), 9 samples were predicted correctly as $0.1 \mathrm{mM}$, but 1 sample was predicted as $8 \mathrm{mM}$. The lda score plots is also given in the supplementary data (Fig. S6). In addition, the proposed system was integrated with a simple and user-friendly mobile application GlucoQuantifier to quantify the glucose concentration. Image is selected from the gallery or capture using the camera then the colored area is cropped and sent to the server that 
runs machine learning classifier via Firebase to quantify the concentration level, the result comes back and it is displayed on the GlucoQuantifier.

In addition, colorimetric assays were performed using $s$-Au/Ag NPs with samples of glucose, galactose, maltose, and xylose to evaluate the specificity of the proposed system. As shown in Fig. 6, the percent accuracy value is significantly higher for glucose compared to galactose, maltose, and xylose. It can be concluded that the $s$ $\mathrm{Au} / \mathrm{Ag}$ NPs are highly specific for distinguishing glucose from other sugars employed in this study. A detailed investigation dealing with the selectivity of the $s$-Au/Ag NPs towards a glucose standard solution in the presence of different interferents such as ascorbic acid, uric acid, dopamine, acetaminophen, L-tyrosine, and sodium chloride is underway. Different synthesis strategies using green reducing agents or substrates will be performed to control the size, shape, and surface charge of the nanoparticles. The effect of the weight ratio of reducing agents to metal precursors and the reaction time on producing the bimetallic nanoparticles with various sizes and dispersity will be investigated. In addition, surface functionalization of the nanoparticles with polymers, biomolecule, surfactants, dendrimers, and small molecules can be developed to improve the stability of the nanoparticles and to prevent their aggregation, and obtain targetspecific probe. Furthermore, the reliabilities of the non-enzymatic sensor will be evaluated by determining the glucose concentration in tears, urine, and interstitial body fluid which enables non-invasive detection.

\section{Conclusion}

Herein, a machine learning approach is proposed for enzyme-free colorimetric glucose detection based on different-sized $\mathrm{Au} / \mathrm{Ag}$ NPs as the GOx mimic using a smartphone. The 
general mechanism of $\mathrm{Au} / \mathrm{Ag}$ NPs for the colorimetric detection relies on the interaction between surface functional groups of the NPs with glucose molecules, and the sizedependent surface charge density of the NPs. In this study, large and small-sized Au/Ag NPs were employed for qualitative and quantitative colorimetric evaluation of assays. A dataset was created with images of assays containing different-sized Au/Ag NPs and glucose solutions at various concentrations to train machine learning classifiers. Moreover, a custom-designed smartphone application (GlucoQuantifier) was developed to communicate with the remote server running machine learning classifiers to determine the glucose concentration of the assay. Among the tested classifiers, 93.63\% classification accuracy was reached with $s-\mathrm{Au} / \mathrm{Ag}$ NPs which demonstrates a broad prospect for quantification of the colorimetric assays. To the best of our knowledge, this study is the first to link machine learning classifiers with $\mathrm{Au} / \mathrm{Ag}$ NPs based on a smartphone application that provides a non-enzymatic quantitative analysis of glucose for rapid and portable on-site surveillance without the need for complex equipment. However, the synthesis of target-specific nanoparticles with high stability and selectivity, and the potential interferences in real samples, are still issues that need to be considered. Besides, illumination conditions, imaging distance/angle, smartphone brands are critical parameters to ensure the robustness of the sensing system. This study can be further extended by training the classifiers with closer concentration levels to improve the classification sensitivity, and employing more sophisticated methodologies including deep learning and transfer learning to increase the classification accuracy.

\section{Acknowledgments}

This research was supported by the Scientific and Technological Research Council of 
Turkey (TUBITAK)-British Council (The Newton-Katip Celebi Fund Institutional Links, Turkey-UK project: 116E934) and the Scientific Research Projects Coordination unit of Izmir Katip Celebi University (Project no. 2018-ÖDL-MÜMF-0021).

\section{References}

1. P. Saeedi, I. Petersohn, P. Salpea, B. Malanda, S. Karuranga, N. Unwin, S. Colagiuri,

L. Guariguata, A. A. Motala, K. Ogurtsova, J Diabetes research clinical practice, 2019, $157,107843$.

2. L. Guariguata, D. R. Whiting, I. Hambleton, J. Beagley, U. Linnenkamp, J. E. Shaw, $J$ Diabetes research clinical practice, 2014, 103, 137-149.

3. C. Chen, Q. Xie, D. Yang, H. Xiao, Y. Fu, Y. Tan, S. Yao, J Rsc Advances, 2013, 3, 4473-4491.

4. M.-S. Steiner, A. Duerkop, O. S. Wolfbeis, J Chemical Society Reviews, 2011, 40, 4805-4839.

5. E. Ryckeboer, R. Bockstaele, M. Vanslembrouck, R. Baets, J Biomedical optics express, 2014, $5,1636-1648$.

6. J. Solanki, O. P. Choudhary, P. Sen, J. T. Andrews, J Review of Scientific Instruments, 2013, 84, 073114.

7. L. Jin, L. Shang, S. Guo, Y. Fang, D. Wen, L. Wang, J. Yin, S. Dong, \%J Biosensors Bioelectronics, 2011, 26, 1965-1969.

8. H. V. Hsieh, Z. A. Pfeiffer, T. J. Amiss, D. B. Sherman, J. B. Pitner, J Biosensors Bioelectronics, 2004, 19, 653-660.

9. M. Guo, L. Wei, Y. Qu, F. Zeng, C. Yuan, J Materials Letters, 2018, 213, 174-177.

10. M. Tetik, O. Kap, G. Dutta, V. Kilic, D. Moschou, N. Horzum, in Book An enzymefree glucose biosensor based on $\mathrm{CuO}$ nanostructures anchored on flexible printed circuit 
board, ed., ed. by Editor, City, 2020, Chap. Chapter.

11. H. H. Mai, D. H. Tran, E. Janssens, J Microchimica Acta, 2019, 186, 1-10.

12. Z. Huang, J. Yang, L. Zhang, X. Geng, J. Ge, Y. Hu, Z. Li, J Analytical Methods, 2017, 9, 4275-4281.

13. M. S. Kim, J. Lee, H. T. Ahn, M. I. Kim, J. Lee, J Nanoscale, 2020, 12, 1419-1424.

14. M. Kang, H. Zhou, N. Zhao, B. Lv, J CrystEngComm, 2020, 22, 35-43.

15. T. Meng, H. Jia, H. Ye, T. Zeng, X. Yang, H. Wang, Y. Zhang, \%J Journal of colloid interface science, 2020, 560, 1-10.

16. S. Rashtbari, G. Dehghan, M. Amini, J Analytica chimica acta, 2020, 1110, 98-108.

17. T. Li, K. Zhu, S. He, X. Xia, S. Liu, Z. Wang, X. Jiang, J Analyst, 2011, 136, 28932896.

18. Y. Xianyu, J. Sun, Y. Li, Y. Tian, Z. Wang, X. Jiang, J Nanoscale, 2013, 5, 6303-6306.

19. Y. Zhai, J. Li, X. Chu, M. Xu, F. Jin, X. Li, X. Fang, Z. Wei, X. Wang, J Journal of Alloys Compounds, 2016, 672, 600-608.

20. T. Chen, X. Wu, J. Wang, G. Yang, J Nanoscale, 2017, 9, 11806-11813.

21. T. Lin, L. Zhong, Z. Song, L. Guo, H. Wu, Q. Guo, Y. Chen, F. Fu, G. Chen, J Biosensors Bioelectronics, 2014, 62, 302-307.

22. A. Scandurra, F. Ruffino, S. Sanzaro, M. G. Grimaldi, J Sensors Actuators B: Chemical, 2019, 301, 127113.

23. S. Tabassum, S. Naz, A. Nisar, H. Sun, S. Karim, M. Khan, S. Shahzada, A. ur Rahman, M. Ahmad, J New Journal of Chemistry, 2019, 43, 18925-18934.

24. S. Samuei, J. Fakkar, Z. Rezvani, A. Shomali, B. Habibi, J Analytical biochemistry, 2017, 521, 31-39.

25. K. Promsuwan, N. Kachatong, W. Limbut, J Electrochimica Acta, 2019, 320, 134621. 26. M. Li, L. Fang, H. Zhou, F. Wu, Y. Lu, H. Luo, Y. Zhang, B. Hu, J Applied Surface 
Science, 2019, 495, 143554.

27. F. Wang, Y. Zhang, W. Liang, L. Chen, Y. Li, X. He, J Sensors Actuators B: Chemical, 2018, 273, 41-47.

28. L. Su, W. Qin, H. Zhang, Z. U. Rahman, C. Ren, S. Ma, X. Chen, J Biosensors Bioelectronics, 2015, 63, 384-391.

29. C. Ding, Y. Yan, D. Xiang, C. Zhang, Y. Xian, J Microchimica Acta, 2016, 183, 625631.

30. H. Wei, E. Wang, J Analytical Chemistry, 2008, 80, 2250-2254.

31. X. Zhao, H. Zhao, L. Yan, N. Li, J. Shi, C. Jiang, J Critical reviews in analytical chemistry, 2020, 50, 97-110.

32. K. Yoshimura, M. Maeda, N. Kamiya, T. Zako, J Analytical Sciences, 2020, 20 SCP12.

33. T.-C. Lim, Nanosensors: theory and applications in industry, healthcare and defense. Editor, CRC Press, 2016.

34. S. Nishat, F. R. Awan, S. Z. Bajwa, J Analytical Sciences, 2018, 18 R001.

35. Y. Gao, Y. Wu, J. Di, J Spectrochimica Acta Part A: Molecular Biomolecular Spectroscopy, 2017, 173, 207-212.

36. S. Adnan, N. H. Kalwar, M. W. Abbas, R. A. Soomro, M. A. Saand, F. R. Awan, A. Avci, E. Pehlivan, S. Bajwa, J SN Applied Sciences, 2019, 1, 144.

37. P. A. Nair, K. Sreenivasan, J Analytical Methods, 2016, 8, 2082-2087.

38. Y.-P. Li, L. Jiang, T. Zhang, M. Lin, D.-B. Tian, H. Huang, J Chinese Chemical Letters, 2014, $25,77-79$.

39. T. Golcez, V. Kilic, M. Sen, J Analytical Sciences, 2020, $20 \mathrm{P} 262$.

40. Ö. Kap, V. Kilic, J. G. Hardy, N. Horzum, J Analyst, 2021.

41. V. Kiliç, M. Şen, in Book Smartphone-based Colorimetric Analysis for the Detection of H 2 O 2 Using a uPAD, ed., ed. by Editor, IEEE, City, 2019, Chap. Chapter, pp. 1-4. 
42. T. Gölcez, V. Kiliç, M. Şen, in Book Integration of a Smartphone Application with a uPAD for Rapid Colorimetric Detection of Glucose, ed., ed. by Editor, IEEE, City, 2019, Chap. Chapter, pp. 1-4.

43. Y. Jung, J. Kim, O. Awofeso, H. Kim, F. Regnier, E. Bae, Applied optics, 2015, 54, 9183-9189.

44. L. Shen, J. A. Hagen, I. Papautsky, Lab on a Chip, 2012, 12, 4240-4243.

45. S. Sumriddetchkajorn, K. Chaitavon, Y. Intaravanne, J Sensors Actuators B: Chemical, 2013, 182, 592-597.

46. Y. Intaravanne, S. Sumriddetchkajorn, J Computers Electronics in Agriculture 2015, $116,228-233$.

47. Ö. B. Mercan, V. Doğan, V. K1lıç, in Book Time Series Analysis based Machine Learning Classification for Blood Sugar Levels, ed., ed. by Editor, IEEE, City, 2020, Chap. Chapter, pp. 1-4.

48. Ö. B. Mercan, V. Kılıç, M. Şen, J Sensors Actuators B: Chemical, 2021, 329, 129037. 49. H. Kim, O. Awofeso, S. Choi, Y. Jung, E. Bae, Applied Optics, 2017, 56, 84-92.

50. A. K. Yetisen, J. Martinez-Hurtado, A. Garcia-Melendrez, F. da Cruz Vasconcellos, C. R. Lowe, Sensors and Actuators B: Chemical, 2014, 196, 156-160.

51. B. Berg, B. Cortazar, D. Tseng, H. Ozkan, S. Feng, Q. Wei, R. Y.-L. Chan, J. Burbano, Q. Farooqui, M. Lewinski, J ACS nano, 2015, 9, 7857-7866.

52. G. A. Helfer, V. S. Magnus, F. C. Böck, A. Teichmann, M. F. Ferrão, A. B. d. Costa, $J$ Journal of the Brazilian Chemical Society, 2017, 28, 328-335.

53. Ö. B. Mercan, V. Kılıç, in Book Fuzzy classifier based colorimetric quantification using a smartphone, ed., ed. by Editor, Springer, City, 2020, Chap. Chapter, pp. 12761283

54. J. Turkevich, P. C. Stevenson, J. Hillier, J Discussions of the Faraday Society, 1951, 
$11,55-75$.

55. G. Topcu, T. Guner, E. Inci, M. M. Demir, J Sensors Actuators A: Physical, 2019, 295, 503-511.

56. T. Parnklang, C. Lertvachirapaiboon, P. Pienpinijtham, K. Wongravee, C. Thammacharoen, S. Ekgasit, J RSC advances, 2013, 3, 12886-12894.

57. Z. Fan, Y. Xu, D. Zhang, J IEEE Transactions on Neural Networks, 2011, 22, 11191132.

58. L. Breiman, J Machine learning, 1996, 24, 123-140.

59. L. Breiman, J Machine learning, 2001, 45, 5-32.

60. A. Y. Mutlu, V. K1lıç, in Book Machine learning based smartphone spectrometer for harmful dyes detection in water, ed., ed. by Editor, IEEE, City, 2018, Chap. Chapter, pp. $1-4$.

61. V. Kılıç, J Avrupa Bilim ve Teknoloji Dergisi, 289-294.

62. G. James, D. Witten, T. Hastie, R. Tibshirani, An introduction to statistical learning. Editor, Springer, 2013, Vol. 112.

63. A. Rohiman, I. Anshori, A. Surawijaya, I. Idris, in Book Study of colloidal gold synthesis using Turkevich method, ed., ed. by Editor, American Institute of Physics, City, 2011, Vol. 1415, Chap. Chapter, pp. 39-42.

64. A. O. Dada, F. A. Adekola, O. S. Adeyemi, O. M. Bello, A. C. Oluwaseun, O. J. Awakan, F.-A. A. Grace, in Silver Nanoparticles-Fabrication, Characterization and Applications, IntechOpen, 2018.

65. K. M. Koczkur, S. Mourdikoudis, L. Polavarapu, S. E. Skrabalak, J Dalton Transactions, 2015, 44, 17883-17905.

66. B. M. Rajam, P. Ramasamy, U. Mahalingam, J Acta Chimica Slovenica, 2017, 64, 186-192. 
67. S. Smitha, K. Nissamudeen, D. Philip, K. Gopchandran, J Spectrochimica Acta Part A: Molecular Biomolecular Spectroscopy, 2008, 71, 186-190.

68. C.-C. Chang, C.-P. Chen, T.-H. Wu, C.-H. Yang, C.-W. Lin, C.-Y. Chen, J Nanomaterials, 2019, 9, 861.

69. L. Mikac, M. Ivanda, M. Gotić, T. Mihelj, L. Horvat, J Journal of nanoparticle research, 2014, 16, 1-13.

70. P. Suchomel, L. Kvitek, R. Prucek, A. Panacek, A. Halder, S. Vajda, R. Zboril, J Scientific reports, 2018, $8,1-11$. 
Table 1 The synthesized Au and Ag NPs with the corresponding particle size estimated from SEM micrographs

\begin{tabular}{ccccc}
\hline NPs & Reducing agent & Stabilizer & Synthesis conditions & Diameter (nm) \\
\hline$\ell$ - Au NPs & $\mathrm{NaBH}_{4}$ & $\mathrm{Na}_{3} \mathrm{C}_{6} \mathrm{H}_{5} \mathrm{O}_{7}$ & Room temperature & $30 \pm 12$ \\
$s$-Au NPs & $\mathrm{NaBH}_{4}$ & $\mathrm{Na}_{3} \mathrm{C}_{6} \mathrm{H}_{5} \mathrm{O}_{7}$ & Heated under reflux & $16 \pm 4$ \\
& & and $\mathrm{PVP}$ & & \\
$\ell$-Ag NPs & $\mathrm{NaBH}_{4}$ & $\mathrm{Na}_{3} \mathrm{C}_{6} \mathrm{H}_{5} \mathrm{O}_{7}$ & Room temperature & $25 \pm 5$ \\
$s$-Ag NPs & $\mathrm{NaBH}_{4}$ & $\mathrm{Starch}_{4}$ & Heated up to boiling & $12 \pm 2$ \\
\hline
\end{tabular}


Table 2 Classification results on the $\ell$ and $s$-Au/Ag NPs datasets

\begin{tabular}{|l|c|c|c|}
\cline { 2 - 4 } \multicolumn{1}{c|}{} & \multicolumn{2}{c|}{ Accuracy (\%) } \\
\cline { 2 - 4 } & Classifiers & $\ell$-Au/Ag NPs & s-Au/Ag NPs \\
\hline \multirow{2}{*}{$\mathbf{1}^{\text {st }}$ day } & LDA & 88.18 & 90.00 \\
\cline { 2 - 4 } & BC & 80.00 & 85.45 \\
\cline { 2 - 4 } & RF & 79.09 & 79.09 \\
\hline \multirow{2}{*}{$\mathbf{2}^{\text {nd }}$ day } & LDA & 90.00 & 93.63 \\
\cline { 2 - 4 } & BC & 80.90 & 80.00 \\
\cline { 2 - 4 } & RF & 76.36 & 88.18 \\
\hline
\end{tabular}


Table 3 Classification report on the $\ell-\mathrm{Au} / \mathrm{Ag}$ NPs and $s$-Au/Ag NPs dataset

\begin{tabular}{|l|c|c|c|c|c|c|}
\cline { 2 - 7 } & \multicolumn{3}{c|}{ l-Au/Ag NPs } & \multicolumn{3}{c|}{ s-Au/Ag NPs } \\
\cline { 2 - 7 } & precision & recall & f1-score & precision & recall & f1-score \\
\hline $\mathbf{0 ~} \mathbf{~ m M}$ & 0.90 & 0.90 & 0.90 & 1.00 & 1.00 & 1.00 \\
\hline $\mathbf{0 . 5} \mathbf{~ m M}$ & 0.89 & 0.80 & 0.84 & 0.91 & 1.00 & 0.95 \\
\hline $\mathbf{1 . 0} \mathbf{~ m M}$ & 0.70 & 0.70 & 0.70 & 1.00 & 1.00 & 1.00 \\
\hline $\mathbf{2 . 0} \mathbf{~ m M}$ & 1.00 & 1.00 & 1.00 & 1.00 & 1.00 & 1.00 \\
\hline $\mathbf{3 . 0} \mathbf{~ m M}$ & 0.75 & 0.90 & 0.82 & 0.90 & 0.90 & 0.90 \\
\hline $\mathbf{4 . 0} \mathbf{~ m M}$ & 0.89 & 0.80 & 0.84 & 1.00 & 1.00 & 1.00 \\
\hline $\mathbf{5 . 0} \mathbf{~ m M}$ & 1.00 & 1.00 & 1.00 & 1.00 & 0.60 & 0.75 \\
\hline $\mathbf{6 . 0} \mathbf{~ m M}$ & 1.00 & 1.00 & 1.00 & 0.91 & 1.00 & 0.95 \\
\hline $\mathbf{7 . 0} \mathbf{~ m M}$ & 0.83 & 1.00 & 0.91 & 0.69 & 0.90 & 0.78 \\
\hline $\mathbf{8 . 0} \mathbf{~ m M}$ & 1.00 & 1.00 & 1.00 & 1.00 & 0.90 & 0.95 \\
\hline $\mathbf{9 . 0} \mathbf{~ m M}$ & 1.00 & 0.80 & 0.89 & 1.00 & 1.00 & 1.00 \\
\hline Average & 0.91 & 0.90 & 0.90 & 0.95 & 0.94 & 0.93 \\
\hline
\end{tabular}




\section{Figure Captions}

Fig. 1 (a) Laboratory type photo studio, (b) Color change of $s$-Au/Ag NPs at different glucose concentrations

Fig. 2 Steps of colorimetric glucose detection with GlucoQuantifier. The homepage of the GlucoQuantifier is shown in (a) where the user can view the image from the gallery or take a new image with the smartphone camera to display on the screen as in (b). The adjustable cropbox is used in (c) to extract the ROI given in (d). The ROI is uploaded to the cloud for the quantification of glucose in (e) and the result is displayed in (f).

Fig. 3 UV-Vis absorption spectra of the (a) $\ell$-Au NPs and $\ell$-Ag NPs, (b) mixed solution of $\ell-\mathrm{Au} / \mathrm{Ag}$ NPs at different volume ratios, SEM micrographs of the (c) $\ell$-Au NPs and (d) $\ell$-Ag NPs, the particle size distribution of the (e) $\ell$-Au NPs and (f) $\ell$-Ag NPs

Fig. 4 UV-Vis absorption spectrum of the (a) $s$-Au NPs and $s$-Ag NPs, SEM micrographs of the (b) $s$-Au NPs and (c) $s$-Ag NPs, the particle size distribution of the (d) $s$-Au NPs and (e) $s$-Ag NPs $\}$

Fig. 5 Confusion matrix of LDA on the (a) $\ell$-Au NPs and $\ell$-Ag NPs dataset and (b) $s$-Au NPs and $s$-Ag NPs dataset

Fig. 6 Specificity investigation of the $s$-Au/Ag NPs system for the detection of glucose. The percent accuracy values in response to $3 \mathrm{mM}$ samples of different analytes. 


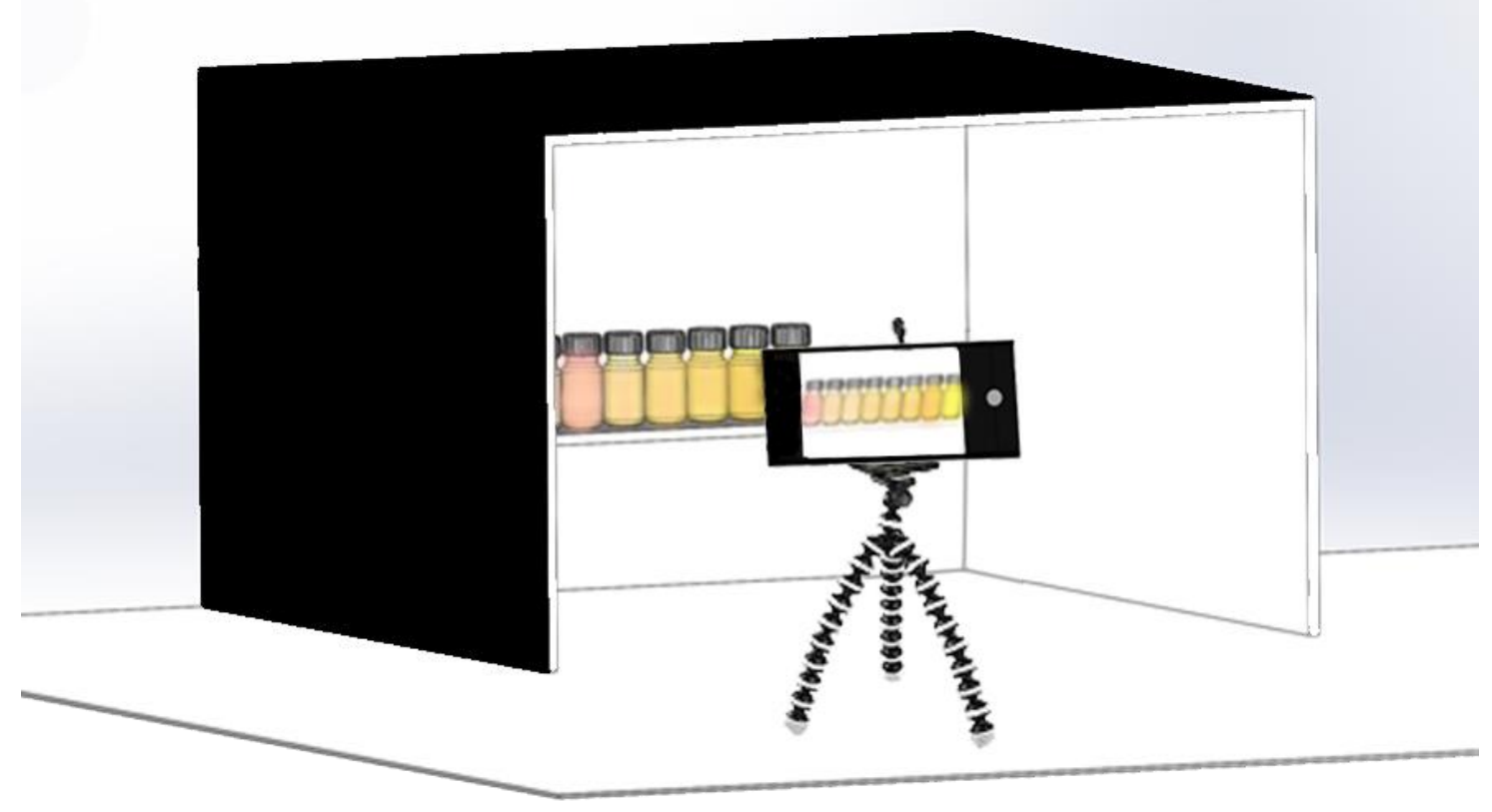

(a)

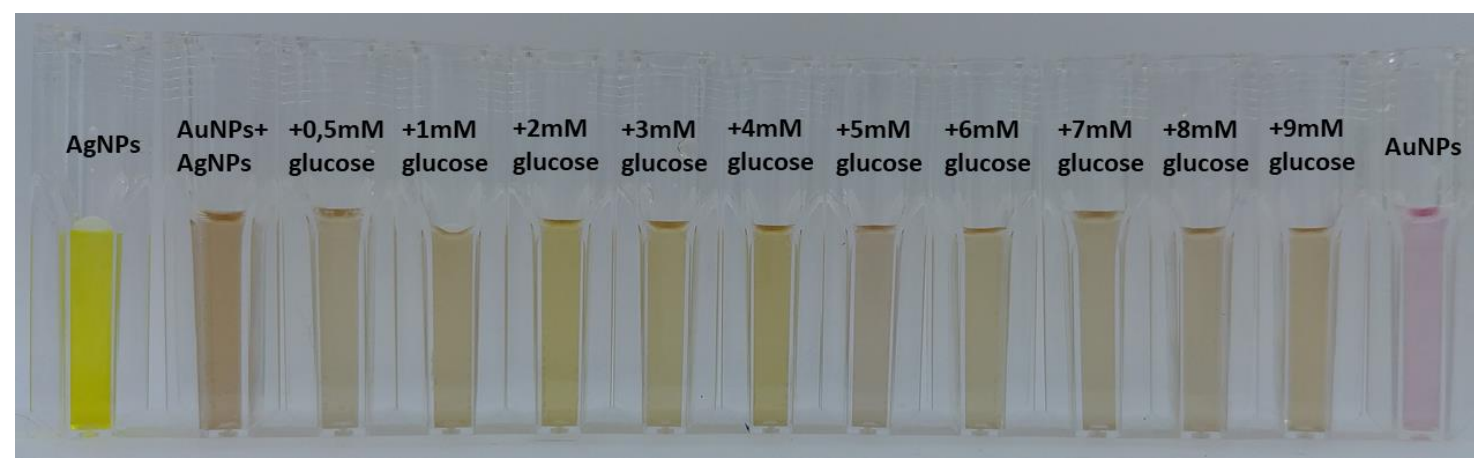

(b)

Fig. 1 


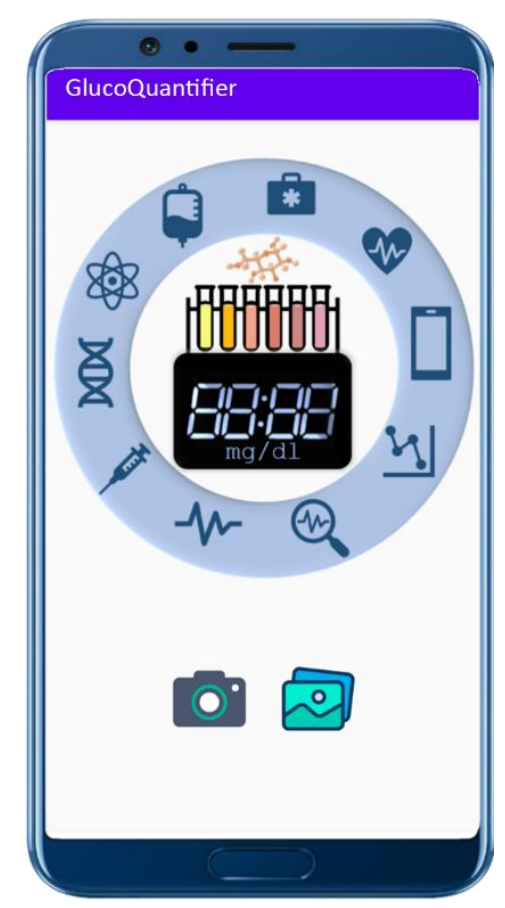

(a)

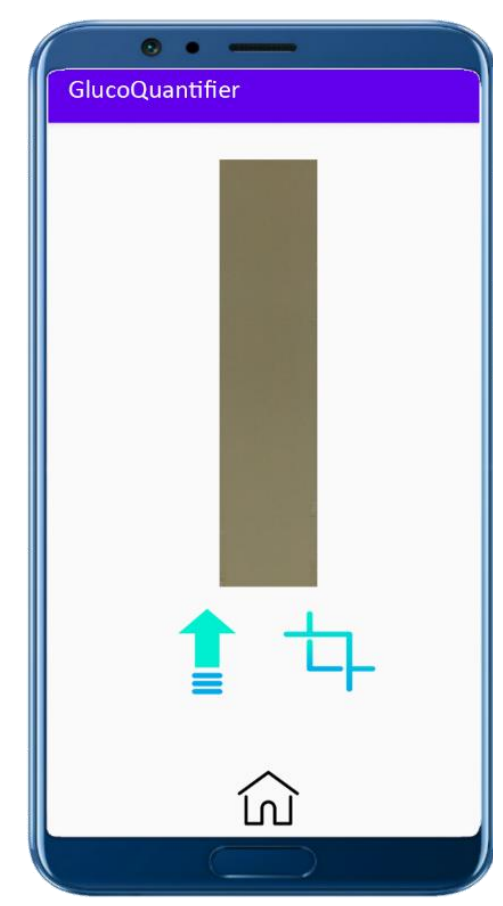

(d)

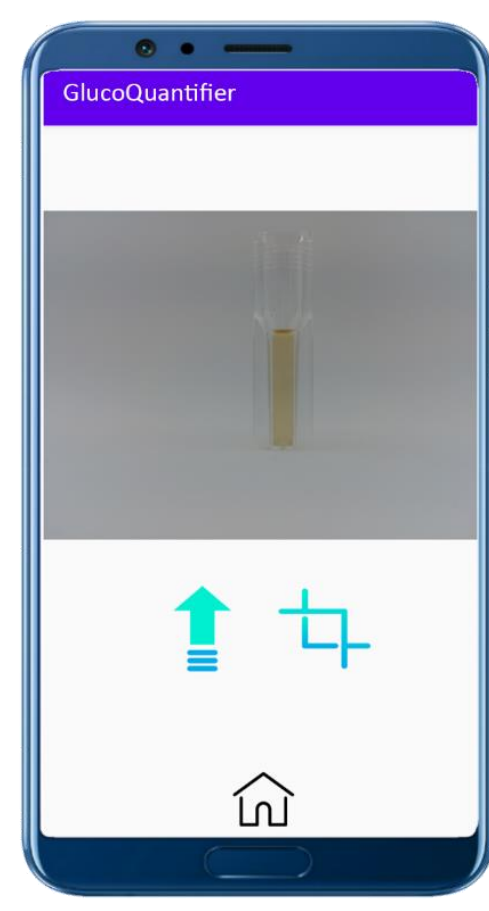

(b)

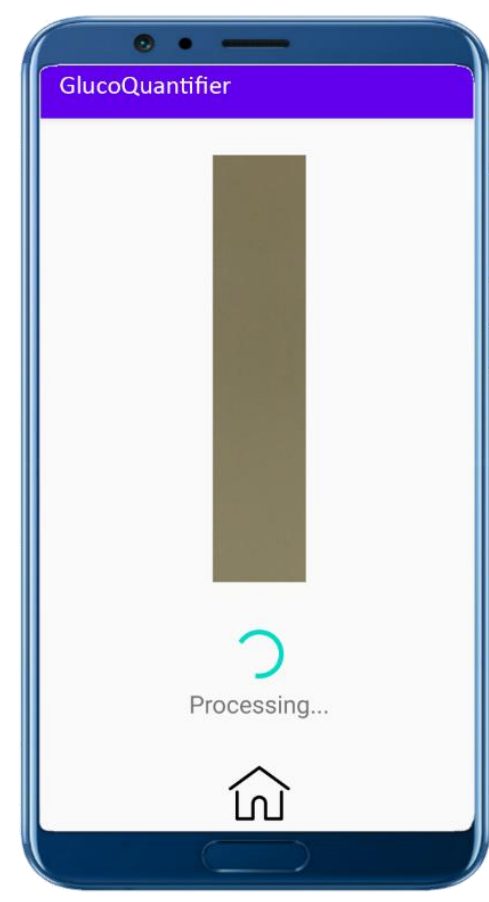

(e)

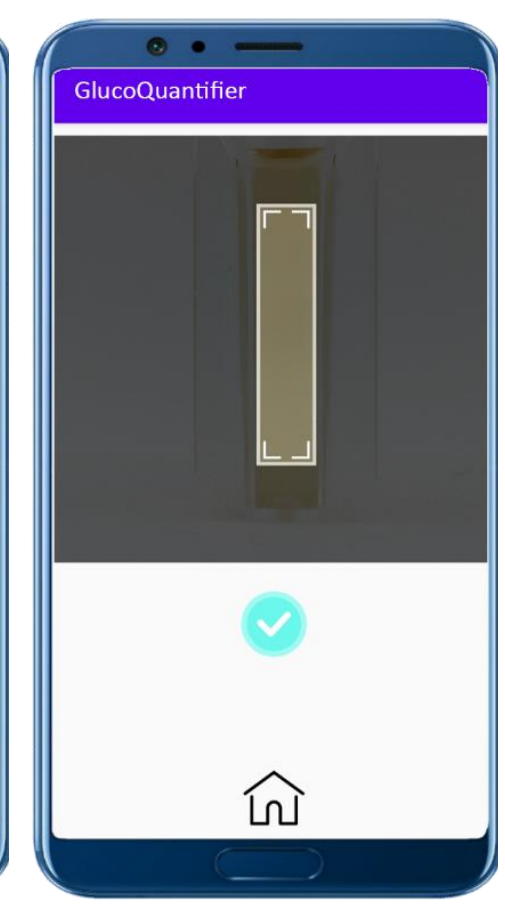

(c)

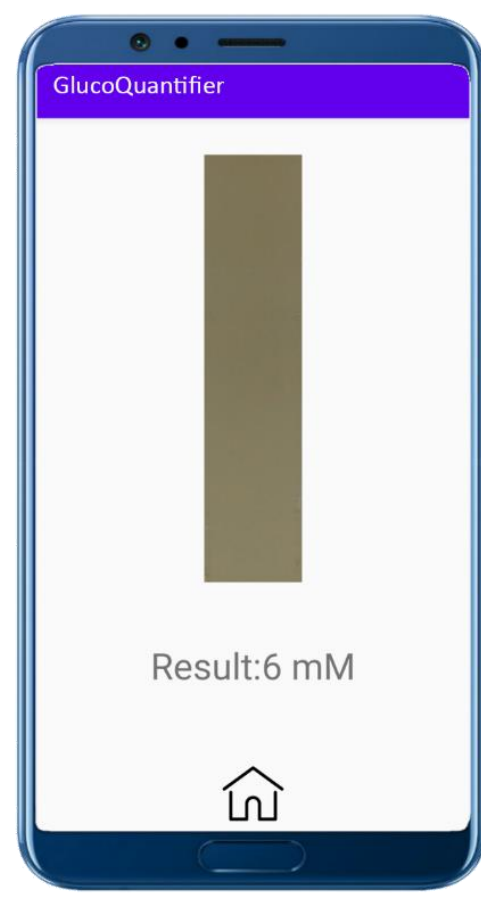

(f)

Fig. 2 


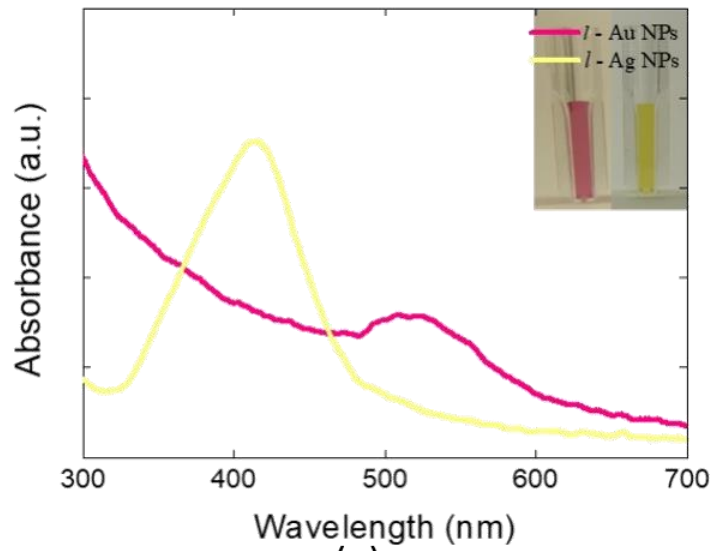

(a)

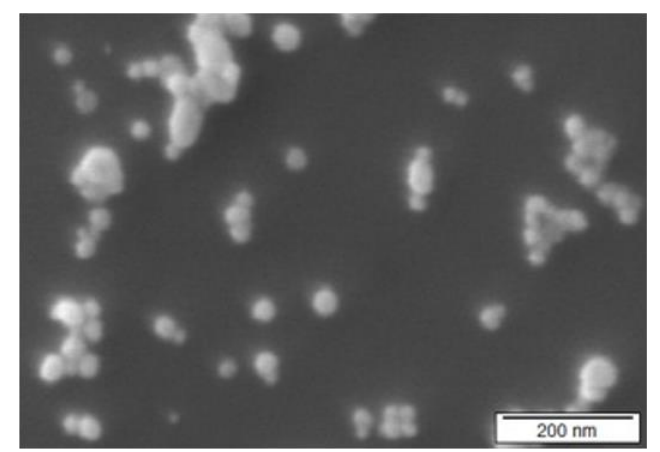

(c)

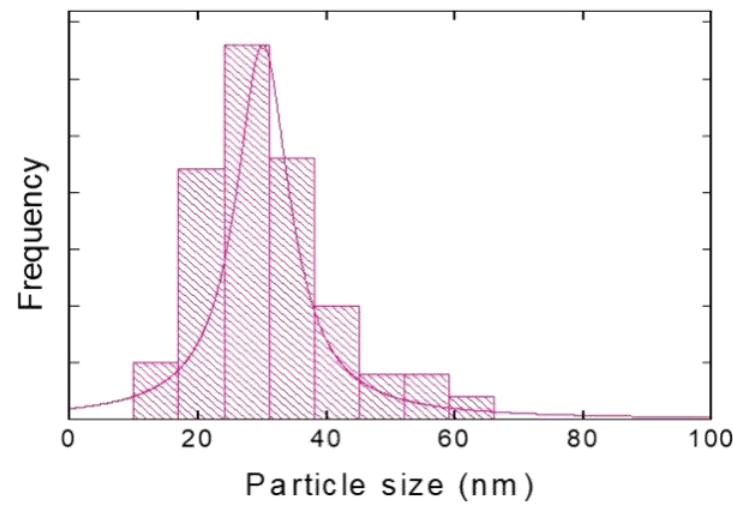

(e)

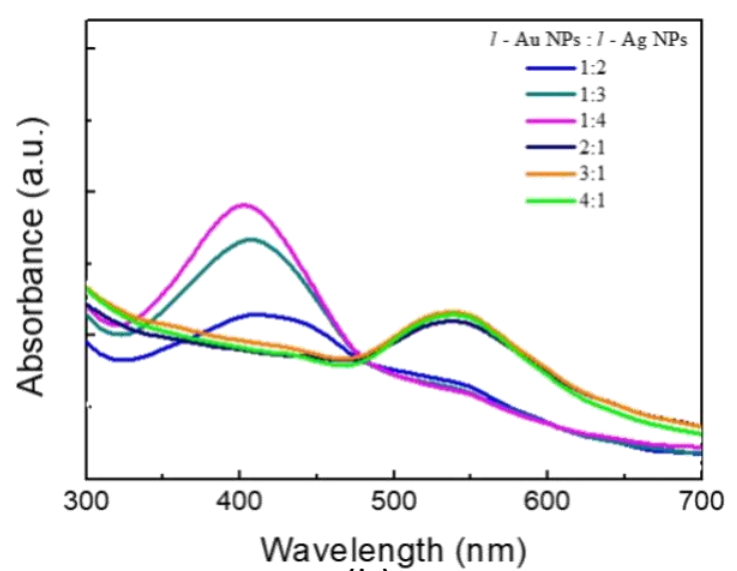

(b)

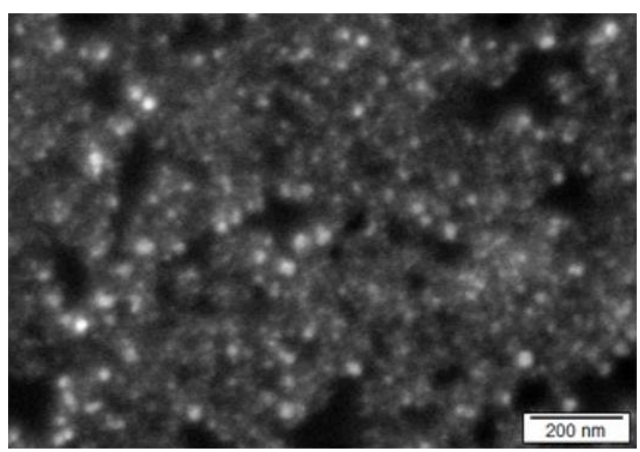

(d)

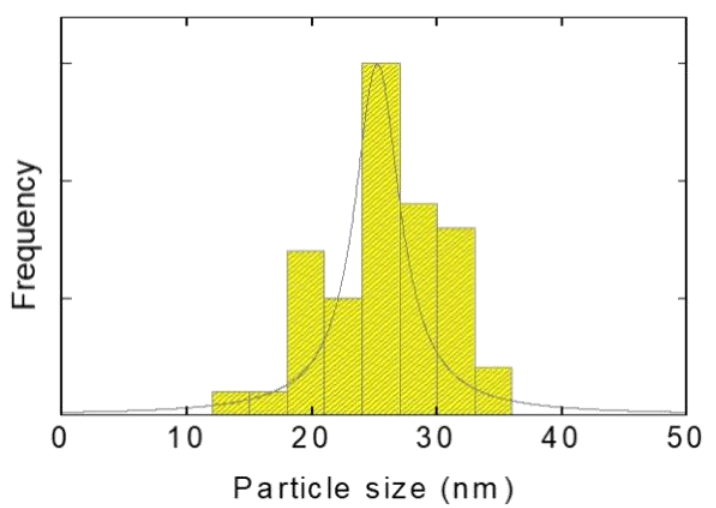

(f)

Fig. 3 


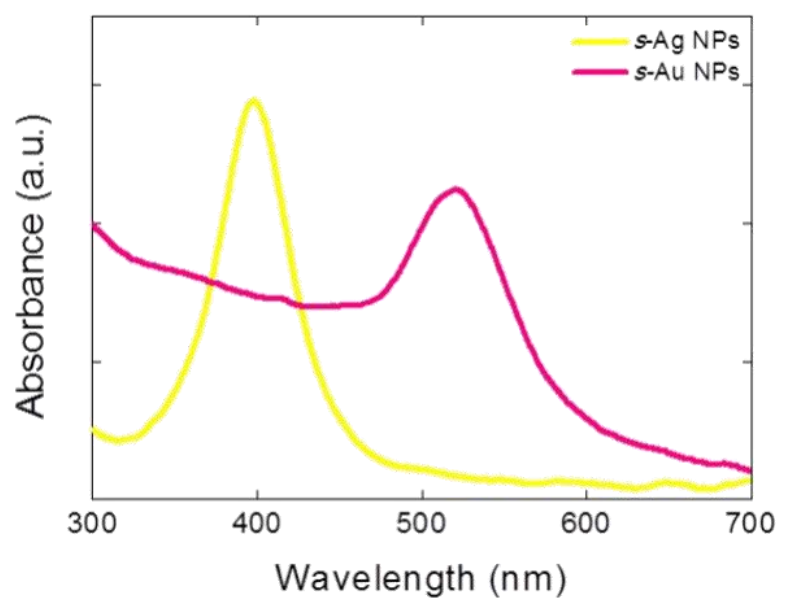

(a)

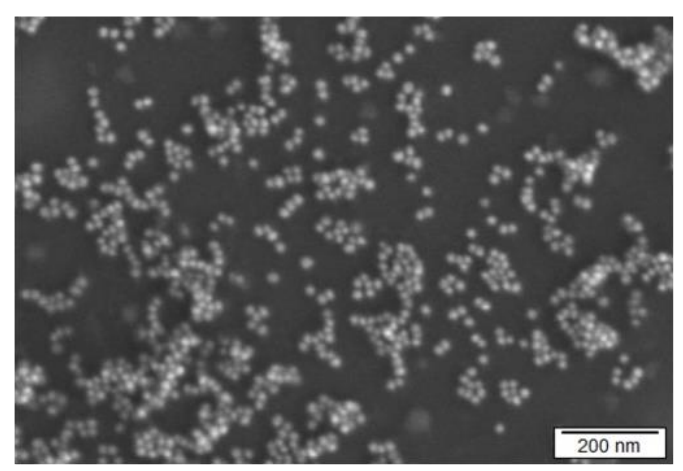

(b)

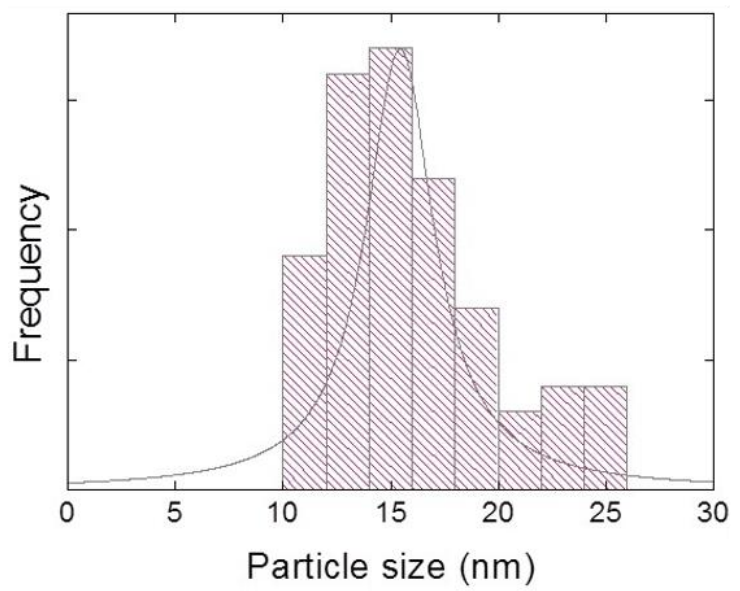

(d)

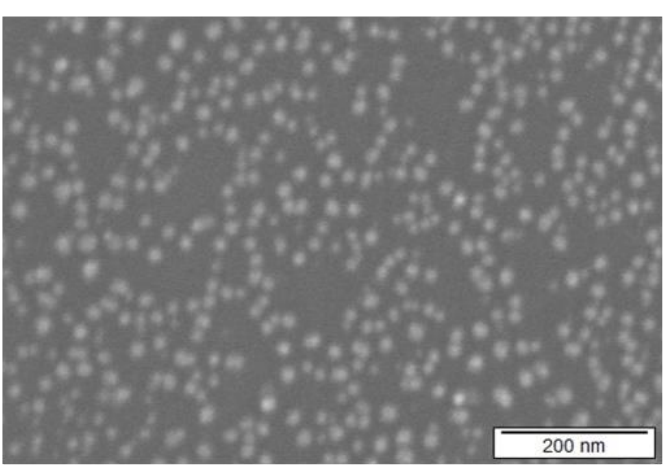

(c)

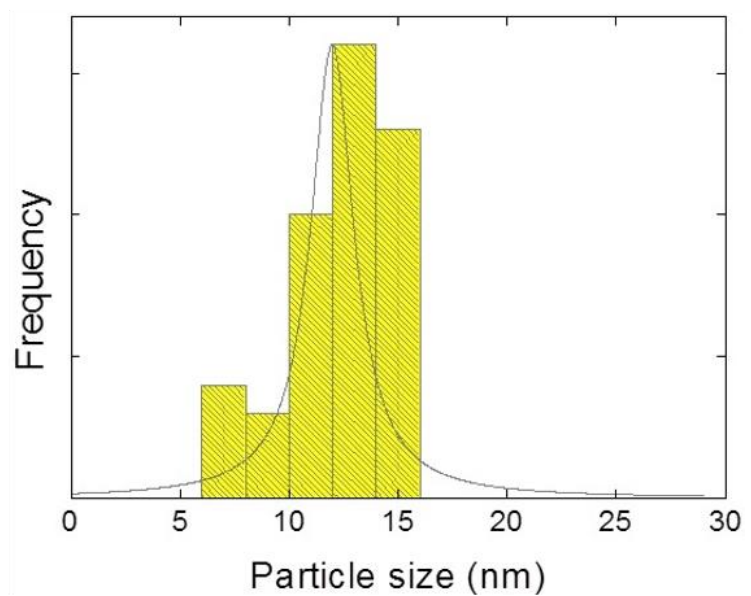

(e)

Fig. 4 


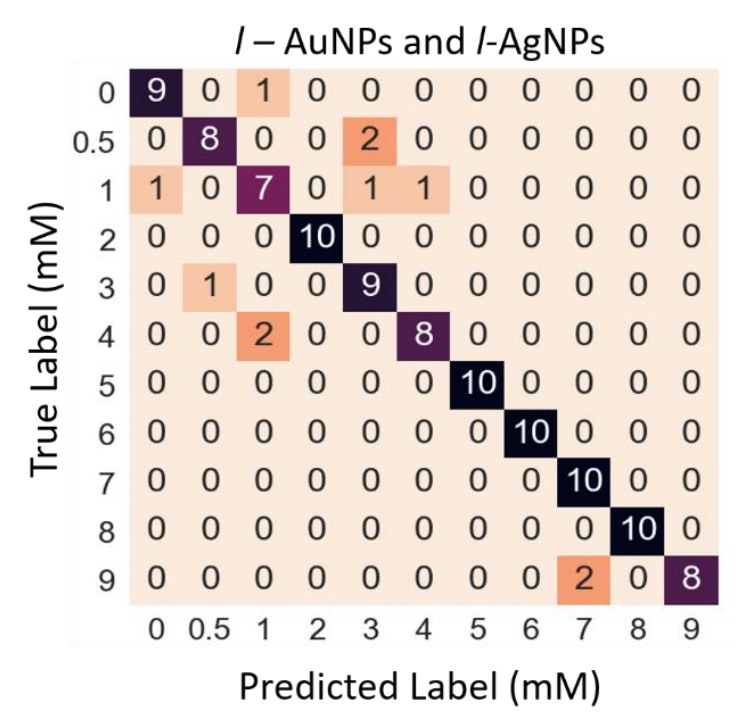

(a)

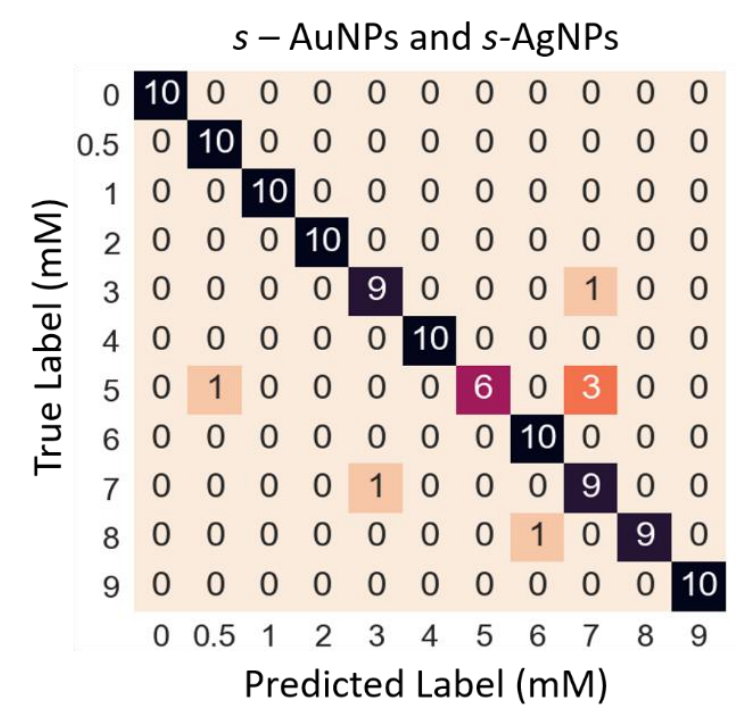

(b)

Fig. 5 


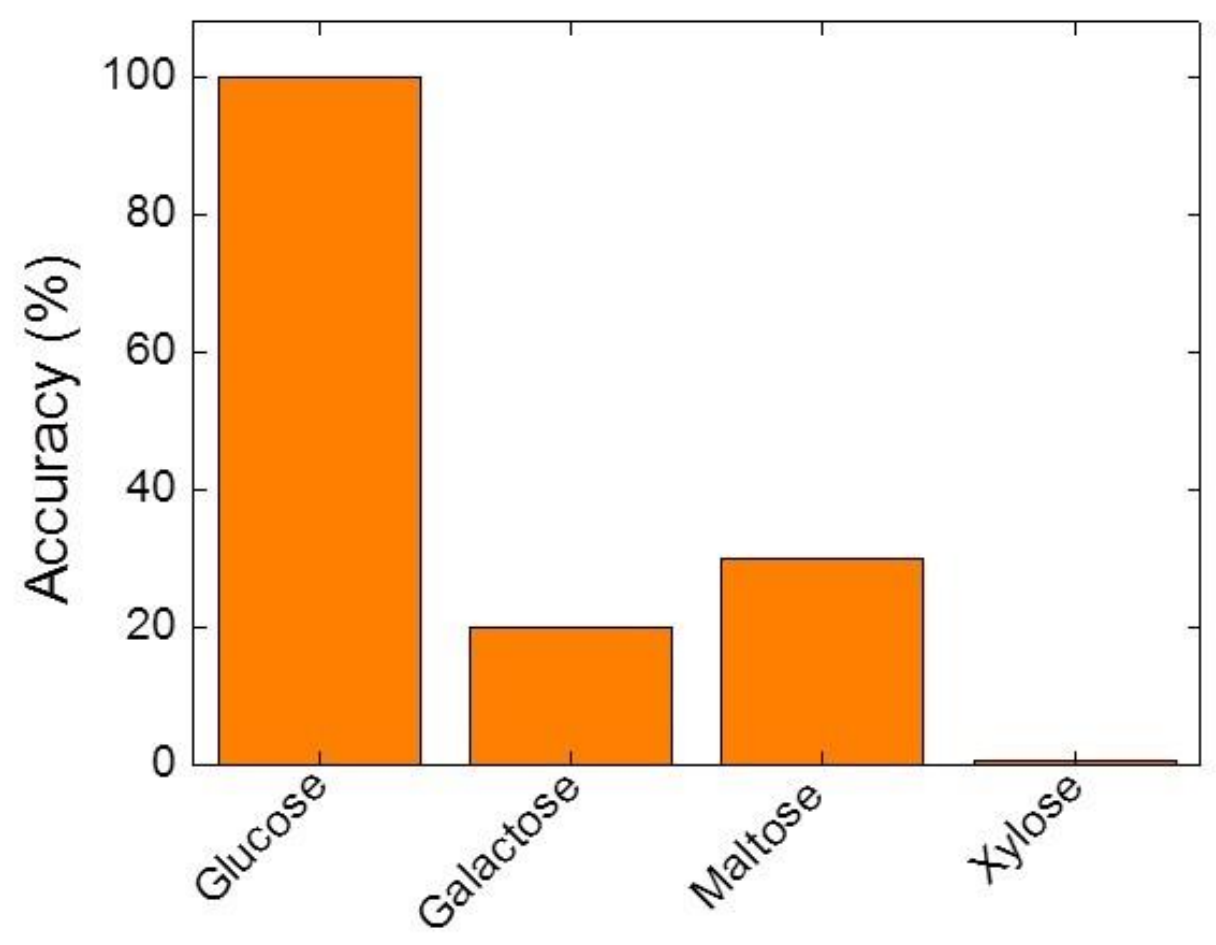

Fig. 6 


\section{Graphical Index}

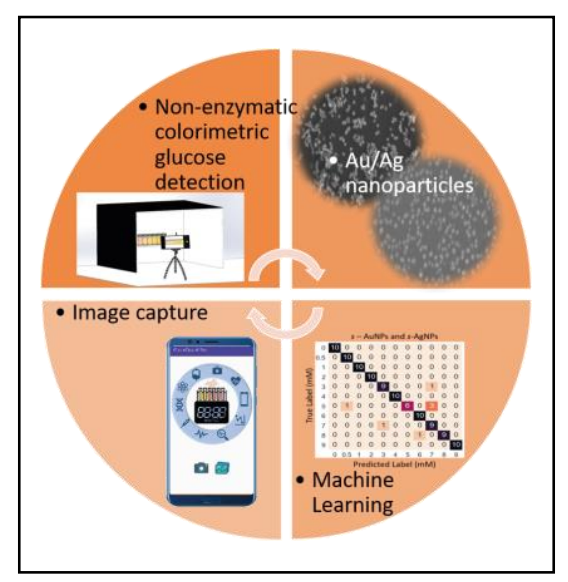

